\title{
Efficacy of Arthrocentesis with Injection of Hyaluronic Acid in The Treatment of Internal Derangement of Temporomandibular Joint
}

\author{
Pramila Shakya' ${ }^{1}$ Quazi Billur Rahman'2, Shakhawat Hossain ${ }^{3}$, Mahmuda Akhter ${ }^{3}$, Md. Wares Uddin ${ }^{3}$ \\ ${ }^{1}$ MS Student, ${ }^{2}$ Associate Professor, ${ }^{3}$ Assistant Professor, Department of Oral \& Maxillofacial Surgery Faculty of Dentistry, BSMMU, Dhaka
}

\begin{abstract}
:
This cross-sectional study was designed to find out the effect of arthrocentesis with injection hyaluronic acid in the treatment of internal derangement of temporomandibular joint (TMJ ID) for the restoration of TMJ function. The study included 25 patients, aged 13 to 50 years with symptoms of TMJ pain and clicking during function and limited mouth opening. TMJ ID was assessed with clinical examination and conformed with MRI. Arthrocentesis was done with insertion of two 21gauze needles in the upper joint compartment and joint lavaged with $200 \mathrm{ml}$ of ringer's lactate solution and at the end $1 \mathrm{ml}$ of hyaluronic acid was injected. Intensity of TMJ pain and clicking was assessed using visual analog scale, maximum mouth opening, lateral jaw movement and protrusion were assessed with millimeter scale. All the parameters were measured before the procedure, immediately after the procedure then after 2 weeks, 1 month , 3 months and 6 months procedure. During 6 months follow-up, clinical examination and comparison of the results showed 84\% reduction in TMJ pain, 92\% improvement in mouth opening and clicking disappear in $80 \%$ of patients.
\end{abstract}

Key words: TMJ pain, clicking, internal derangement, arthrocentesis and hyaluronic acid.

[BSMMU J 2010; 3(1): 18-22]

\section{Introduction:}

Temporomandibular joint disorders (TMDs) are one of the most misdiagnosed and mistreated maladies in the medical practice. TMDs are not life threatening but they may strongly affect the quality of life. Painful temporomandibular joint function disturbs day to day life and body image. These problems sometimes become so significant that they may impair person emotional stability. ${ }^{1}$

Epidemiological study has shown that at-least 33 percent of American population suffer from this problem. ${ }^{2}$ The males to females ratio seeking care of TMD is $4: 1$. Accordingly, analgesics directed at the TMD are among the top selling over the counter medicine in America. ${ }^{3}$

Internal derangement of the temporomandibular joint (TMJ ID) is one of the most frequent cause of TMD. TMJ ID is defined as a progressive disorder which usually starts as clicking associated with normal opening (anterior disc displacement with reduction ), to a stage where clicking gradually ceases but restricted mouth opening ensues(closed lock). ${ }^{4}$ TMJ ID is usually characterized by pain, clicking, deviated jaw and limitation of jaw movement. ${ }^{5}$ Many conservative approach have been proposed throughout the years among which are occlusal splint therapy, physiotherapy, complimentary medication

Address for Correspondence: Dr. Pramila Shakya, MS Student, Department of Oral \& Maxillofacial Surgery Faculty of Dentistry, BSMMU, Dhaka and occlusal treatment. ${ }^{6}$ In the past treatment of TMJ ID that did not respond to conservative measure was surgical recontouring and repositioning of the disc. ${ }^{7}$

Nitzan et al. ${ }^{4}$ first describe the TMJ arthrocentesis as the simplest and effective minimal invasive modalities for treatment of TMJ closed lock. Irrigation of the superior joint compartment will results in creation of hydraulic pressure, which will release the displaced disc and thereby re-establish normal maximal mouth opening. With the intend to extend the indication of TMJ arthrocentesis, a technique of providing the injection of hyaluronic acid at the end of articular lavage was purposed. ${ }^{6}$ Intra-articular corticosteroid are occasionally injected to alleviate the intracapsular inflammation. ${ }^{8}$ Unfortunately, intra articular corticosteroid injection has an unpredictable prognosis and also cause local side effect on the joint. ${ }^{9}$ Hyaluronic acid has been proposed as an alternative therapeutic agent with same therapeutic effect, that improve and restore normal lubrication in joint, provides nutrition to the vascular articulating disc and stabilize the joint. ${ }^{10,11}$

The purpose of this cross-sectional study was to find out the effect of arthrocentes with injection of hyaluronic acid in the treatment of TMJ ID for the full restoration of TMJ function.

\section{Materials and methods:}

The study involved 25 patients (14 females and 11 males ; age range 13 to 50 years; mean $25 \pm 8.4$ years) who were referred to department of Oral and Maxillofacial Surgery, 
BSMMU, Bangladesh from January 2008 to December 2009. The diagnostic groups had anterior disc displacement with reduction (ADDR) and anterior disc displacement without reduction (ADDwOR) proved by MRI. Patients with symptoms of TMJ pain, clicking, and mouth opening less than $35 \mathrm{~mm}$ and lateral jaw movement less than $5 \mathrm{~mm}$ for duration from 1 month to 1 year who failed to respond to conservative measures were included in the study. Patients responding to conservative therapy, giving history of hard tissue injuries, ankylosis and medically unfit for treatment were excluded from the study.

All patients were given conservative therapy for 2 weeks before arthrocentesis, which included non-steroidal anti inflammatory drugs (NSAID), muscle relaxant, anxiolytics, soft diet, occlusal splint and lifestyle modification.

Each patient completed a questionnaire evaluating TMJ pain, joint clicking, and maximum mouth opening. TMJ pain assessed using visual analog scale (VAS) in a $10 \mathrm{~cm}$ line with one end labeled with no pain and another end with severe pain. Maximum mouth opening (MMO), lateral excursion, protrusion were measured with millimeter scale and clicking sound assessed by palpating the joint during opening and closing of mouth.

Informed written consent were taken from the patients and arthrocentesis was done in the Oral and Maxillofacial department outdoor under local anaesthesia .

\section{Procedure of Arthrocentesis:}

Patient was placed in supine position with head in opposite direction. The pre-auricular area of the affected site was prepared aseptically with betadin solution. Auriculotemporal nerve was blocked through the skin just anterior to the junction of the tragus and ear-lobe. The needle was advanced behind the posterior aspect of the condyle in anteromedial direction to depth of $1 \mathrm{~cm}$ and 1.5 $\mathrm{ml}$ of 1:80000 lignocain with adrenalin was deposited.

A line was drawn from mid tragus of ear to lateral canthus of eye. First point was marked $12 \mathrm{~mm}$ anterior from tragus and $2 \mathrm{~mm}$ downward in the tragal canthal line. Second point was marked $22 \mathrm{~mm}$ anterior and $6 \mathrm{~mm}$ downward in the same line. A 21 gauze needle was inserted from first point upward, inward and forward at 45 degree angle to all plane corresponding posterior joint compartment and approximately $2 \mathrm{ml}$ ringers lactate solution deposited to distend the upper joint compartment (UJC). A second needle was inserted in second point backward, upward and inward to correspond the area of articular eminence to establish a free flow of irrigating solution from the UJC. The joint was irrigated with at-least $200 \mathrm{ml}$ of ringer's lactate solution. During the procedure patients were advised to close and open the mouth to distend the joint and then at the end of lavage $1 \mathrm{ml}$ of hyaluronic acid was injected. Both needles were removed and all the parameters for TMJ functions were measured and data recorded on follow up chart. Patients were advised on soft diet and NSAID prescribed for 2 weeks.

Post-operatively follow up were given on the 2 weeks, 1 month, 3 months and 6 months with all the parameters for TMJ functions measured with same technique. The collected data were analyzed and comparison made between pre-operative values and post operative follow up values. The criteria for success was no pain or minimal pain( pain less than 2), clicking absent and maximum mouth opening more than $38 \mathrm{~mm}$. Data were processed and statistically analyzed using SPSS (statistical package for social science) version 16 .

\section{Results:}

In the study 25 patients were included (14 females and 11 males), with their mean age $25 \pm 8.4$ yrs (range from $13-50$ years). 12 patients had ADDwR and 13 patient had ADDwOR. All patients were given same modalities of treatment and followed- up for 6 months. Pre treatment, immediately after treatment and at follow up data were collected and entered into computer carefully and meticulously for the analysis in SPSS version 16. The evaluation between different follow up was done by paired ' $t$ ' test and mcnemar chi square test. The result was considered significant if $\mathrm{p}$ value was $<0.05$.

The subjective data elicited from VAS scores showed significant reduction in pain from mean $6.8 \pm 2.02$ to $2.1 \pm 1$ at 6 months follow up. Maximal mouth opening improves from mean $28.4 \pm 4.68 \mathrm{~mm}$ to mean $41.2 \pm 1.99 \mathrm{~mm}$ at 6 months follow up. Lateral excursion improved from mean 5.0 \pm 2.09 $\mathrm{mm}$ to mean $6.8 \pm 1.0 \mathrm{~mm}$. Similarly, TMJ clicking disappear significantly.

The final outcome of the study showed $84 \%$ reduction in pain, 92\% improvement in MMO and clicking absent in $80 \%$ of patients.

Table I

Age distribution of the patients $(n=25)$

\begin{tabular}{lcc}
\hline Age in years & Number of patients & Percentage \\
\hline$\leq 20$ & 7 & 28.0 \\
$20-30$ & 15 & 60.0 \\
$31-40$ & 2 & 8.0 \\
$41-50$ & 1 & 4.0 \\
Mean \pm SD & 25.0 & \pm 8.4 \\
Range (min-max) & $(13$ & $-50)$ \\
\hline
\end{tabular}

The mean age of the patients was 25.0 years with standard deviation \pm 8.4 years with ranged from 13 to 50 years. Maximum patients were in 20-30 year age group. 


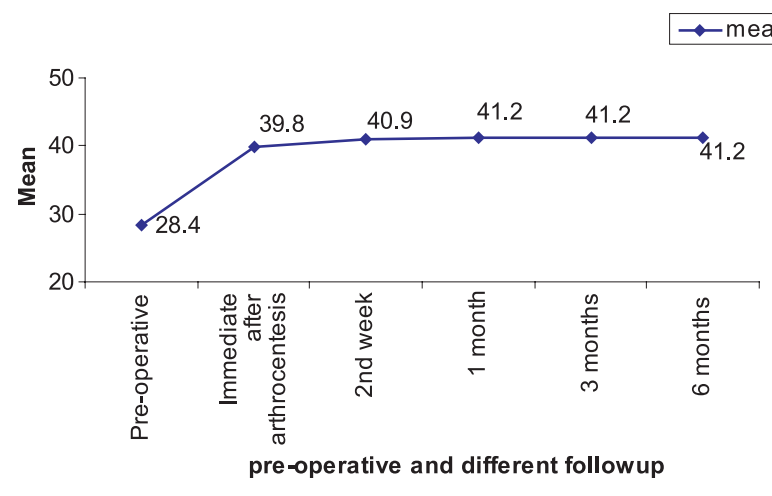

Fig.-1: Line diagram showing the outcome of MMO at different follow-up

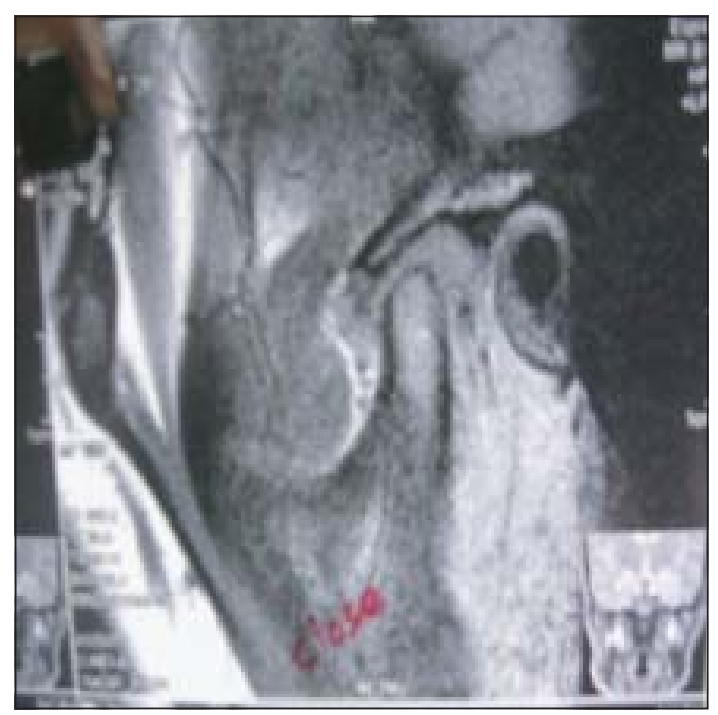

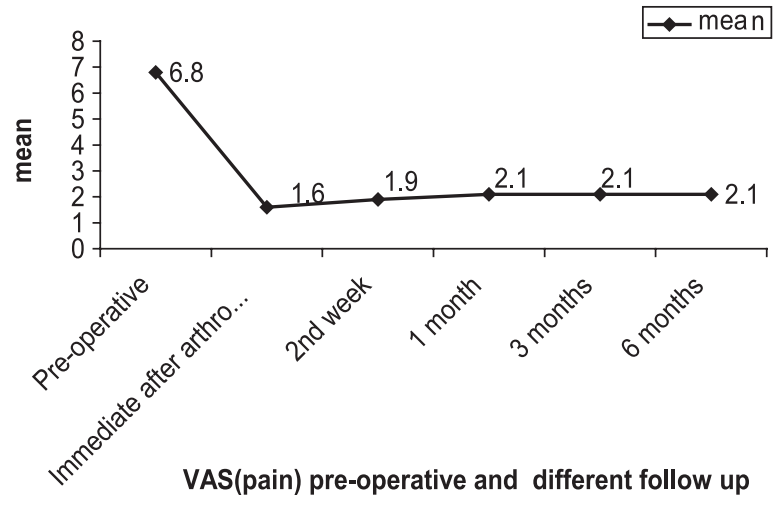

Fig.-2: Line diagram showing the outcome of VAS (pain) at different follow-up

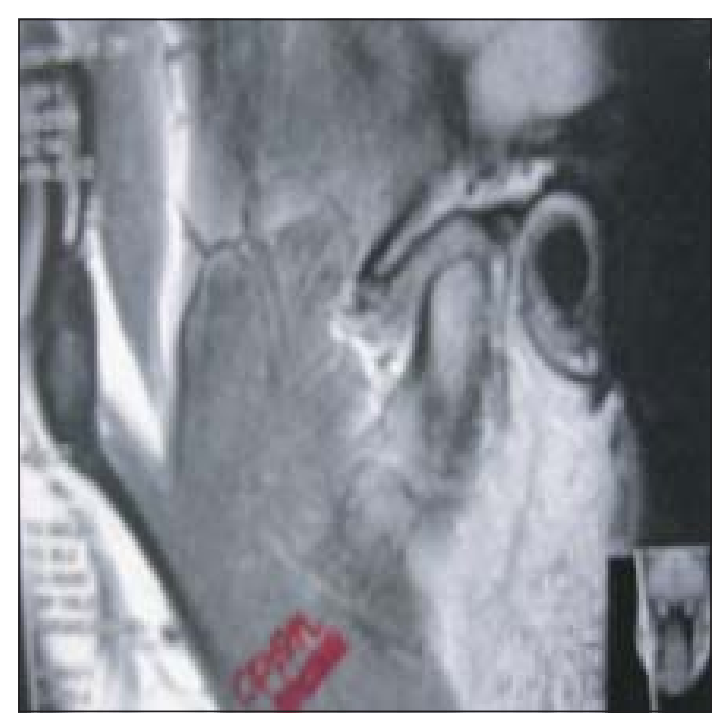

MRI showing anterior disc displacement in close and open view

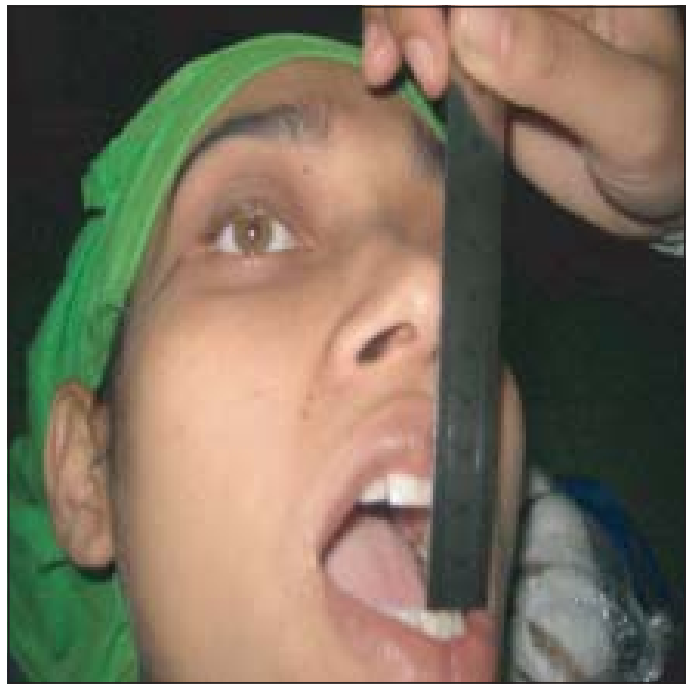

Pre operative oral opening 20mm

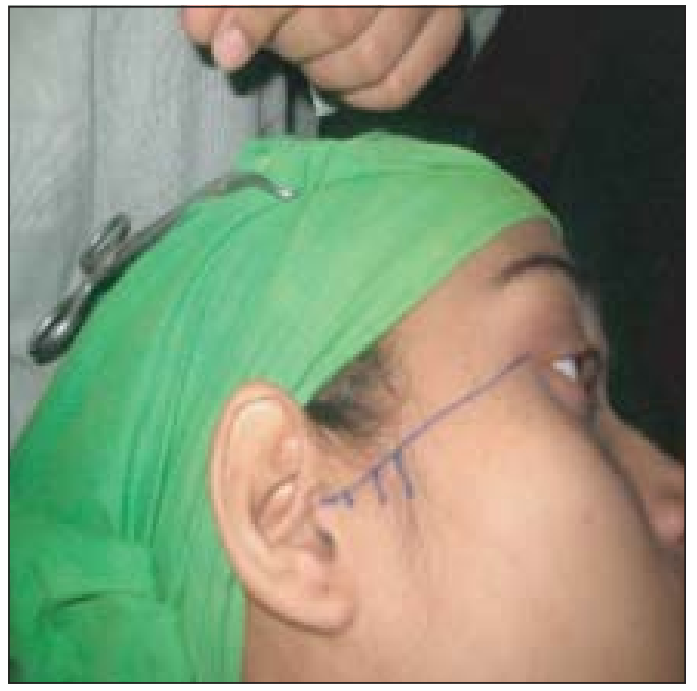

Marking points for arthrocentesis 


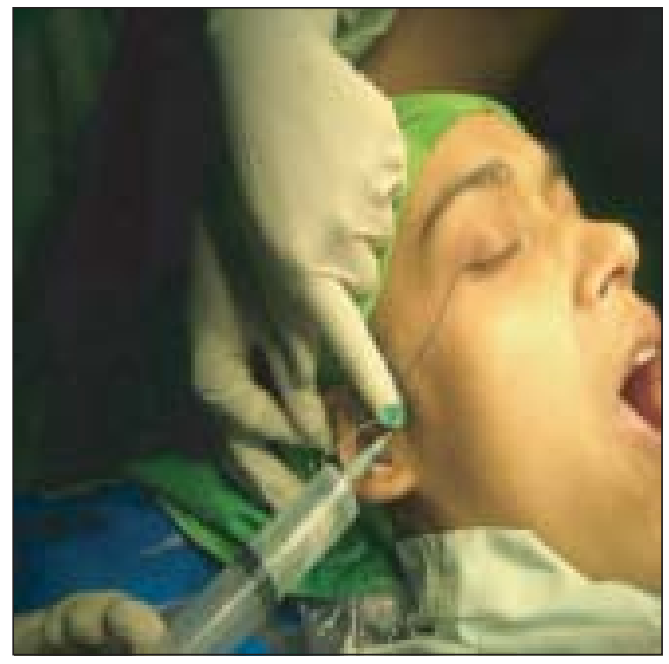

Lavage of upper joint compartment

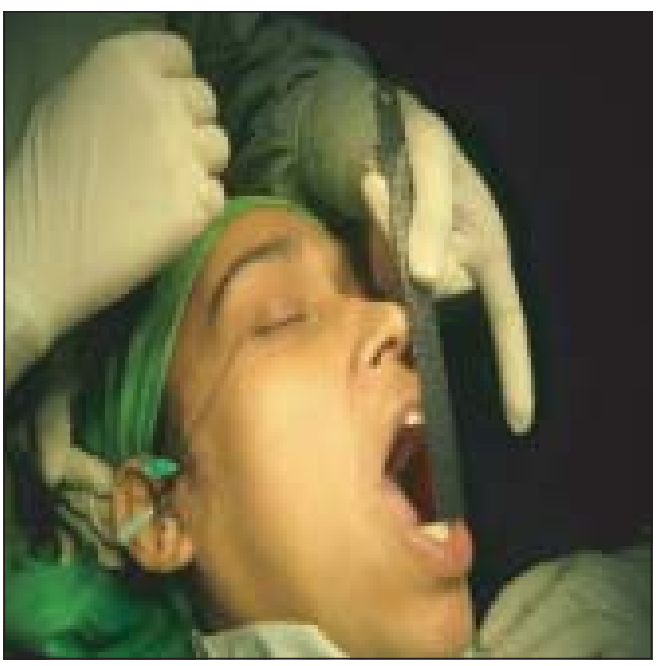

Oral opening after athrocentesis 40mm

\section{Discussion:}

The effectiveness of arthrocentesis with injection of hyaluronic acid in treatment of internal derangement of the TMJ in this study was based on 3 clinical parameters: increase in $\mathrm{MMO}$, reduction in pain and clicking sound during function.

Many researcher and clinician have reported the results of series of patient treated with arthrocentesis and they are uniformly positive. Nitzan et al. ${ }^{4}$ describe the high success rate of $91 \%$ in 17 cases treated with arthrocentesis only. Frost et al. ${ }^{12}$ reviewed 40 treated cases and concluded that the procedure is quite reliable and satisfy both the clinician and patient in acute TMJ locking but frequently unsatisfactory in chronic cases. Hosaka et al. ${ }^{13}$ in 3 months up follow up study reported $74 \%$ success rate . Murakami et al. ${ }^{14}$ in a 6 months follow up reported $70 \%$ success rate. Al-Belasy \& Dolwick ${ }^{15}$ reported in their review study that no medication was used for intra articular injection in four studies, steroid was used in 14 studies and hyaluronic acid was used in 2 studies. Alpaslan \& Alpaslan ${ }^{16}$ found that patient with internal derangement benefited from arthrocentesis with or without injection of hyaluronic acid in terms of relief of pain. But they reported that arthrocentesis with hyaluronic acid injection seems to be superior to arthrocentesis alone.

Alpaslan \& Alpaslan, ${ }^{16}$ showed improvement in MMO in patient treated with arthrocentesis with hyaluronic acid was from mean $34.31 \pm 9.81 \mathrm{~mm}$ to $42.35 \pm 6.05$. similarly Yeung et al. ${ }^{17}$ in 27 patient in 6 month duration with same technique showed improvement in MMO fron mean $38.2 \mathrm{~mm}$ to $39.8 \mathrm{~mm}$. In this study, improvement in $\mathrm{MMO}$ was from mean $28.4 \pm 4.68 \mathrm{~mm}$ to $41.2 \pm 1.99 \mathrm{~mm}$ (94\%) and the result was significent $(\mathrm{p}=0.001)$ and similar to the previous study.

Alpaslan \& Alpaslan, ${ }^{16}$ showed improvement in lateral jaw movement from $8.16 \pm 2.10 \mathrm{~mm}$ to $9.14 \pm 1.23 \mathrm{~mm}$ and showed intensity of pain decreased significantely $(p<0.05)$. Yeung et al, ${ }^{17}$ showed improvement in lateral jaw movement from mean $7.4 \mathrm{~mm}$ to $8.2 \mathrm{~mm}$ and decreased in pain in VAS from mean $4.2 \mathrm{~mm}$ to mean $2.6 \mathrm{~mm}$. In this study, lateral jaw movement increases from mean $5.0 \pm 2.09 \mathrm{~mm}$ to $6.80 \pm 1.00 \mathrm{~mm}$ and the result was significient $(\mathrm{p}=0.001)$. similarly, pain decreased in VAS from mean $6.8 \pm 2.02$ to $1.6 \pm 0.64$.

Yeung et al. ${ }^{17}$ showed reduction in joint clicking was significant in 6 month duration $(\mathrm{p}<0.05)$. Sato et al. ${ }^{18}$ in retrospective cohort study with clicking present in 59 patients showed absent of clicking in 52 patients(88\%). In our study, clicking reduced in 20 patients (80\%) and result co-relate with previous study.

\section{Conclusion:}

Arthrocentesis with injection of hyaluronic acid in the treatment of TMJ internal derangement is least invasive procedure with fewest complications. The study showed satisfactory results in restoration of TMJ functions in patients with TMJ ID, who were refractory to the conservative methods and psycologically depressed due to lack of proper treatment.

Thus, it may be the preferred treatment for the patient suffering with TMJ ID who were refractory to conservative methods. 


\section{References:}

1. Malik N. Textbook of oral and maxillofacial surgery. $2^{\text {nd }}$.ed. New Delhi: Jaypee Brothers Medical Publishers (P) Ltd 2002, pp. 240-256.

2. Okeson JP. Orofacial pain: Guidelines for assessment, diagnosis, and management. Chicago: Quintessence Publishing Co 1996.

3. Prater ME, Bailey BJ, Quinn FB. A source of information. In: Temporomandibular joint disorders. The university of texas branch 1998.

4. Nitzan DW, Dolwick MF, Martinez GA. Temporomandibular joint arthrocentesis: a simplified treatment for severe, limited mouth opening. J Oral Maxillofac Surg 1991;49 :1163-1167.

5. Emshoff R, Rudisch A. Are internal derangement and osteoarthrosis linked to changes in clinical outcome measures of arthrocentesis of the temporomandibular joint. J Oral Maxillofac Surg 2003; 61:1162-1167.

6. Nardini GL, Stifano M, Brombin C, Salmaso L, Manfredini D. A one year series of arthrocentesis with hyaluronic acid injection for temporomandibular joint osteoarthritis. Oral surg Oral Med Oral Pathol Oral Radiol Endod 2007 ;103: e14e22.

7. Dimitroulis G, Dolwick MF, Martinez A. Temporomandibular joint arthrocentesis and lavage for the treatment of closed lock: a follow up study. Br J Oral Maxillofac Surg 1995; 33:2326.

8. Dimitroulis G, Dolwick MF. Temporomandibular disorders. Part 3: Surgical treatment . Aust Dent J 1996;44:16-20.

9. Dionne RA. Pharmacological treatments for temporomandibular disorder. Oral Surg Oral Med Oral Pathol Oral Radiol Endod 1997;83:134.

10. Fader, K. W., et al., 1993. Pressurized infusion of sodium hyaluronate for closed lock of the temporomandibular joint. Part.I: A case study. J Craniomandib Prac, vol.11, p.68.
11. Sato S, Oguri S, Yamaguchi K, Kawamura H, Motegi K. Effect of pumping with injection of sodium hyaluronate and the other factors related to outcome in patients with non reducing disc displacement of the temporomandibular joint. Int J Oral Maxillofac Surg 2001; 30: 194-198.

12. Frost DE, Kendell BD. The use of arthrocentesis for the treatment of temporomandibular joint disorders. J Oralmaxillofac Surg 1999;57: 583-587.

13. Hosaka H, Murakami K, Goto K, Lizuka T. Outcome of arthrocentesis for temporomandibular joint with closed lock at 3years follow up. Oral Surg Oral medOral Pathol Oral Radiol Endod 1996;54: 504-4.

14. Murakami KI, Hosaka H, Moriya Y, Segami N, Lizuka T. Short term outcome study for the management of temporomandibular joint closed lock: a comparision of arthrocentesis to nonsurgical therapy and arthroscopic lysis and lavage. Oral Surg Oral Med Oral Pathol Oral Radiol Endod 1995; 80:253-257.

15. Al-Belasy FA, Dolwick MF. Arthrocentesis for the treatment of temporomandibular joint closed lock: a review article. Int J Oral Maxillofac Surg 2007;36:773-782.

16. Alpaslan GH, Alpaslan C. Efficacy of temporomandibular joint arthrocentesis with or without injection of sodium hyaluronate in treatment of internal derangements. J Oral Maxillofac Surg 2001;59: 613-618.

17. Yeung RW. Short term outcome of intra-articular high molecular weight hyaluronic acid injection for non reducing disc displacement of the temporomandibular joint. Oral Surg Oral Med Oral Pathol Oral Radiol Endol 2006;102: 453-61.

18. Sato S, Goto S, Kasahara T, kawamura H \& Motegi K. Effect of pumping with injection of sodium hyaluronate and other factor related to outcome in patients with non reducing disk displacement of the temporomandibular joint. Int J Oral Maxillofac Surg 2001;30: 194-198 